\title{
Subject Index Vol. 72, 1997
}

\section{Acidemia 125}

Alfentanil 133

Amniotic fluid 118

Anoxia 255

Anthropometric characteristics, neonatal 22 Anthropometry 156 Antinociception 28 Antioxidant 71 Antipyrine 133

Antithrombin III 76 Arachidonic acid 165 Asphyxia 42 Autoresuscitation 255

Bile composition 235

flow 235 Bilirubin 71 Birth asphyxia 216 Body fatness 156 Brain 165,187

damage 227 Breathing 293 Bumetanide 265

Catecholamines 125 Cell culture 363 Cerebral hemodynamics 9

- $\quad$ oxygenation 9

Chemiluminescence 102, 142

Chick embryo 165

Chloramphenicol 28

Chronic lung disease 102

Circadian rhythm 94

Coagulation 76

Cocaine 356 Complement 15

- $\quad$ regulatory proteins 15

Cord serum 210

Corticosteroid-binding globulin

192 Cortisol 192 Cytokines 370

Deacetyldiltiazem 51 Development 51 Dexamethasone 175 Dibutyryl-cyclic adenosine monophosphate 305 Diet 22

Dihydrorhodamine 123201 Diltiazem 51 Diuretic 265

Docosahexaenoic acid 32,165 Drug abuse 112

addiction 112

exposure 356

Egg 210 Eicosanoids 62 Endogenous ouabain 337

- ouabain-like substance 345

Endothelium 62

Endotracheal suctioning 9

Epinephrine 125

Erythropoietin 118

Estrogen 314

Extrahepatic tissues 51

Eye 32

Fatty acid(s) 1 
Fentanyl 133

- synthase 305

Fetal blood sampling 279

overgrowth 22

serum 118 Formoterol 305 French population 156 Furosemide 142

therapy 345

Gestational diabetes, screening

22 Glucose-6-phosphate dehydroge-

nase 273 Glutamate 243 Glutathione 273 Glutathione-S-transferase 273 Glycine 243

Growth 156

- $\quad$ hormone 363

Growth-hormone-releasing factor

363 Guinea pig 314

Hair analysis 356 Heart rate 94 Hen age 380 High-resolution gas chroma-

tography 1 Hot-plate test 28 Human milk 1 17-Hydroxyprogesterone 148 Hyperglycemia 255

Hypoxia 42, 62, 243 Hypoxia-ischemia 187,227

IgE 210

IL-2 329

Inspiratory drive 293

Insulin 22,243

Intrauterine environment 314

- $\quad$ growth retardation 181

Intraventricular hemorrhage 76

Ischemia 42,181,243

Isobutyl methylxanthine 305

Lactate 255 Lactic acid 125 Lipids 1,380 Lipophilia 133 Lipoprotein 380 Liver 165,284 Lung development 370

Malonedialdehyde 102 Maternal flow 133 Maternal-fetal transfer 84 Maze 42 Meconium 71, 112

- drug test 112

Metabolism 51

N-Methyl-Z)-aspartate 243

Milk 210

KAHGEÍl C1997SKarger

E-Mail karger@karger.ch Fax + 41613061234 http://www.karger.ch

Basel

393

Mitochondria 284 Morphine 112 -, neonatal guinea pig 293 Myocardial hypertrophy 175

$\mathrm{Na}+, \mathrm{K}+$-ATPase 243 Near-infrared spectroscopy 9 Neonatal sepsis 201 Neonate(s) 265, 356 -, cord blood 273 Neutrophil(s) 15

respiratory burst 201 Newborn(s) 255, 322

infants 345

pig 32

rat 42

Newly hatched chick 380 Nitric oxide $62,216,322$ 
- $\quad$ - synthase inhibition 227

$\mathrm{N} \omega$-Nitro-L-arginine 227

Norepinephrine 125

Oncogenes 329 Opioid withdrawal 293 ß-Oxidation 284 Oxygen need 227

- $\quad$ tension 314

Oxygenation 125

Palmitate 284 Palmitic acid 32 Parturition 192

Perfusion 32 Perinatal rat 187 Peroxisomes 284 Phagocytosis 142 Pharmacokinetics 265 Pig fetus 192 Placenta 84, 363 Placental transfer 133 Plasma cortisol 148 Polymorphonuclear neutrophils

142 Porcine pituitary 363 Pregnancy 293 Premature infant(s) 102,142,

265,337 Prenatal diagnosis, triploidy 279 Preterm baboon 235

infants 9,76

labor 370

neonates 201 Prostaglandin 181 Pulmonary artery, piglet 62

- pressure 227

Rat 118,255

Renal excretory pattern 345

Reperfusion 181

- $\quad$ injury, brain 216

Respiratory distress syndrome

322,370 Retina 32 Retinal pigment epithelium 32

Saturated phosphatidylcholine

370 Secretin 235 Sexual differences 156 Sodium balance 337

- $\quad$ taurocholate 235

Stressed neonates 201

Sufentanil 133

Surfactant 305

Synthase inhibition 216

T-cell receptor 329 Term neonates 201 Thromboxane 181 Triploidy 279 Tritiated water 133

Ubiquinone 71 Ultradian rhythm 94 Uric acid 102 Urinary nitrate 322

- $\quad$ nitrite 322

Uterine blood flow/volume 314

Verapamil 28 Very-low-birth-weight infants 148

Writhing test 28

Zinc 84

- $\quad$ status 84

394

BiolNeonateVol. 72, 1997

Subject Index 